\title{
PERIODIC SOLUTIONS FOR A SYSTEM OF TOTALLY NONLINEAR DYNAMIC EQUATIONS ON TIME SCALE
}

\author{
ERNEST YANKSON
}

Received 17 December, 2014

\begin{abstract}
Let $\mathbb{T}$ be a periodic time scale. We use a reformulated version of Krasnoselskii's fixed point theorem to show that the system of nonlinear neutral dynamic equation with delay

$$
\left.x^{\Delta}(t)=-A(t) H\left(x^{\sigma}(t)\right)+(Q(t, x(t-r(t))))\right)^{\Delta}+G(t, x(t), x(t-r(t))), t \in \mathbb{T},
$$
\end{abstract}

has periodic solutions on the time scale $\mathbb{T}$.

2010 Mathematics Subject Classification: 34K13; 34A34

Keywords: fixed point, totally nonlinear equation, large contraction, periodic solution

\section{INTRODUCTION}

Motivated by the papers $[1-6,10-12]$ and the references therein, we consider the system of dynamic equation

$$
\left.x^{\Delta}(t)=-A(t) H\left(x^{\sigma}(t)\right)+(Q(t, x(t-r(t))))\right)^{\Delta}+G(t, x(t), x(t-r(t))), t \in \mathbb{T},
$$

where $x^{\Delta}(t)$ is $n \times 1$ column vector determined by $\Delta$-derivative components of $x(t)$, $A(t)=\operatorname{diag}\left[a_{1}(t), a_{2}(t), \ldots, a_{n}(t)\right], H: \mathbb{R}^{n} \rightarrow \mathbb{R}^{n}, Q: \mathbb{T} \times \mathbb{R}^{n} \times \mathbb{R}^{n} \rightarrow \mathbb{R}^{n}$, and $G: \mathbb{T} \times \mathbb{R}^{n} \times \mathbb{R}^{n} \rightarrow \mathbb{R}^{n}$

If $n=1$ and $(Q(t, x(t-r(t))))^{\Delta}=c(t) x^{\Delta}(t-r(t))$ then equation (1.1) reduces to the equation considered in [4]. On the other hand, if $n=1$ and $h\left(x^{\sigma}(t)\right)=x^{\sigma}(t)$, then equation (1.1) reduces to the equation considered in [11]. Thus, in this paper we not only generalize the results obtained in [4] and [11] to systems of equations, but even for $n=1$ our results also extends the work of Ardjouni and Djoudi [4] and Kaufmann and Raffoul [11].

We assume in this work that $r: \mathbb{T} \rightarrow \mathbb{R}$ and that $i d-r: \mathbb{T} \rightarrow \mathbb{T}$ is strictly increasing so that the function $x(t-r(t))$ is well defined over $\mathbb{T}$.

Some preliminary material is presented in the next section. In particular, we will provide some facts about the exponential function on time scale and also state a reformulated version of Krasnoselskii's fixed point theorem. Our main results on the existence of periodic solutions for equation (1.1) is presented in Section 3. 


\section{PRELIMINARIES}

We begin this section by giving some definitions introduced by Actici et al. in [6] and Kaufman and Raffoul in [10].

Definition 1. We say that a time scale $\mathbb{T}$ is periodic if there exist a $p>0$ such that if $t \in \mathbb{T}$ then $t \pm p \in \mathbb{T}$. For $\mathbb{T} \neq \mathbb{R}$, the smallest positive $p$ is called the period of the time scale.

For example, the following time scales taken from [10] are periodic.

(1) $\mathbb{T}=\bigcup_{i=-\infty}^{\infty}[2(i-1) h, 2 i h], h>0$ has period $p=2 h$.

(2) $\mathbb{T}=h \mathbb{Z}$ has period $p=h$.

(3) $\mathbb{T}=\mathbb{R}$.

(4) $\mathbb{T}=\left\{t=k-q^{m}: k \in \mathbb{Z}, m \in \mathbb{N}_{0}\right\}$ where, $0<q<1$ has period $p=1$.

As pointed out in [10], all periodic time scales are unbounded above and below.

Definition 2. Let $\mathbb{T} \neq \mathbb{R}$ be a periodic time scale with period $p$. We say that the function $f: \mathbb{T} \rightarrow \mathbb{R}$ is periodic with period $T$ if there exists a natural number $n$ such that $T=n p, f(t \pm T)=f(t)$ for all $t \in \mathbb{T}$ and $T$ is the smallest number such that $f(t \pm T)=f(t)$. If $\mathbb{T}=\mathbb{R}$, we say that $f$ is periodic with period $T>0$ if $T$ is the smallest positive number such that $f(t \pm T)=f(t)$ for all $t \in \mathbb{T}$.

As established in [10], if $\mathbb{T}$ is a periodic time scale with period $p$, then $\sigma(t \pm$ $n p)=\sigma(t) \pm n p$. Consequently, the graininess function $\mu$ satisfies $\mu(t \pm n p)=$ $\sigma(t \pm n p)-(t \pm n p)=\sigma(t)-t=\mu(t)$ and so, is a periodic function with period $p$.

Most of the following definitions, lemmas and theorems can be found in [7, 8]. Our first two theorems concern the composition of two functions. The first theorem is the chain rule on time scales [7, Theorem 1.93].

Theorem 1 (Chain Rule). Assume $v: \mathbb{T} \rightarrow \mathbb{R}$ is strictly increasing and $\tilde{\mathbb{T}}:=v(\mathbb{T})$ is a time scale. Let $w: \tilde{\mathbb{T}} \rightarrow \mathbb{R}$. If $v^{\Delta}(t)$ and $w^{\tilde{\Delta}}(v(t))$ exist for $t \in \mathbb{T}^{\kappa}$, then

$$
(w \circ v)^{\Delta}=\left(w^{\tilde{\Delta}} \circ v\right) v^{\Delta} .
$$

In the sequel we will need to differentiate and integrate functions of the form $f(t-r(t))=f(v(t))$ where, $v(t):=t-r(t)$. Our second theorem is the substitution rule [7, Theorem 1.98].

Theorem 2 (Substitution). Assume $v: \mathbb{T} \rightarrow \mathbb{R}$ is strictly increasing and $\tilde{\mathbb{T}}:=v(\mathbb{T})$ is a time scale. If $f: \mathbb{T} \rightarrow \mathbb{R}$ is an $r d$-continuous function and $v$ is differentiable with $r d$-continuous derivative, then for $a, b \in \mathbb{T}$,

$$
\int_{a}^{b} f(t) v^{\Delta}(t) \Delta t=\int_{v(a)}^{v(b)}\left(f \circ v^{-1}\right)(s) \tilde{\Delta} s .
$$


A function $p: \mathbb{T} \rightarrow \mathbb{R}$ is said to be regressive provided $1+\mu(t) p(t) \neq 0$ for all $t \in \mathbb{T}^{\kappa}$. The set of all regressive rd-continuous functions $f: \mathbb{T} \rightarrow \mathbb{R}$ is denoted by $\mathcal{R}$ while the set $\mathcal{R}^{+}$is given by $\mathcal{R}^{+}=\{f \in \mathcal{R}: 1+\mu(t) f(t)>0$ for all $t \in \mathbb{T}\}$.

Let $p \in \mathcal{R}$ and $\mu(t) \neq 0$ for all $t \in \mathbb{T}$. The exponential function on $\mathbb{T}$ is defined by

$$
e_{p}(t, s)=\exp \left(\int_{s}^{t} \frac{1}{\mu(z)} \log (1+\mu(z) p(z)) \Delta z\right),
$$

It is well known that if $p \in \mathcal{R}^{+}$, then $e_{p}(t, s)>0$ for all $t \in \mathbb{T}$. Also, the exponential function $y(t)=e_{p}(t, s)$ is the solution to the initial value problem $y^{\Delta}=$ $p(t) y, y(s)=1$. Other properties of the exponential function are given in the following lemma, [7, Theorem 2.36].

Lemma 1. Let $p, q \in \mathcal{R}$. Then

(i) $e_{0}(t, s) \equiv 1$ and $e_{p}(t, t) \equiv 1$;

(ii) $e_{p}(\sigma(t), s)=(1+\mu(t) p(t)) e_{p}(t, s)$;

(iii) $\frac{1}{e_{p}(t, s)}=e_{\ominus p}(t, s)$ where, $\ominus p(t)=-\frac{p(t)}{1+\mu(t) p(t)}$;

(iv) $e_{p}(t, s)=\frac{1}{e_{p}(s, t)}=e_{\ominus p}(s, t)$;

(v) $e_{p}(t, s) e_{p}(s, r)=e_{p}(t, r)$;

(vi) $\left(\frac{1}{e_{p}(\cdot, s)}\right)^{\Delta}=-\frac{p(t)}{e_{p}^{\sigma}(\cdot, s)}$.

Lemma 2 ([6]). If $p \in \mathcal{R}^{+}$, then

$$
0<e_{p}(t, s) \leq \exp \left(\int_{s}^{t} p(u) \Delta u\right), \forall t \in \mathbb{T} .
$$

Corollary 1 ([6]). If $p \in \mathcal{R}^{+}$and $p(t)<0$ for all $s \in \mathbb{T}$ with $s \leq t$ we have

$$
0<e_{p}(t, s) \leq \exp \left(\int_{s}^{t} p(u) \Delta u\right)<1, \forall t \in \mathbb{T} .
$$

Lastly in this section, we state Krasnoselskii-Burton's fixed point theorem (see [9]) which is employed in establishing our results.

Theorem 3 (Krasnoselskii-Burton). Let $\mathrm{M}$ be a bounded convex non-empty subset of a Banach space $(S,\|\|$.$) . Suppose that A, B$ map $M$ into $M$ and that

(i) for all $x, y \in \mathbb{M} \Rightarrow A x+B y \in \mathbb{M}$,

(ii) $A$ is continuous and $A M$ is contained in a compact subset of $M$,

(iii) $B$ is a large contraction.

Then there is a $z \in \mathbb{M}$ with $z=A z+B z$.

\section{EXISTENCE OF PERIODIC SOLUTIONS}

Let $T>0, T \in \mathbb{T}$ be fixed and if $\mathbb{T} \neq \mathbb{R}, T=n p$ for some $n \in \mathbb{N}$. By the notation $[a, b]$ we mean

$$
[a, b]=\{t \in \mathbb{T}: a \leq t \leq b\}
$$


unless otherwise specified. The intervals $[a, b),(a, b]$, and $(a, b)$ are defined similarly. Define $P_{T}=\left\{\varphi \in C\left(\mathbb{T}, R^{n}\right): \varphi(t+T)=\varphi(t)\right\}$. Then $P_{T}$ is a Banach space when it is endowed with the usual linear structure as well as the norm

$$
\|x\|=\sum_{j=1}^{n}\left|x_{j}\right|_{0}, \text { for } x=\left(x_{1}, x_{2}, \ldots, x_{n}\right) \in P_{T},
$$

where

$$
\left|x_{j}\right|_{0}=\sup _{t \in[0, T]}|x(t)|, j=1, \ldots, n .
$$

Also, define the set

$$
\mathbb{M}=\left\{\phi \in \mathbb{P}_{\mathbb{T}}:\|\phi\| \leq L \text { with }\left|\phi_{j}\right|_{0} \leq \frac{L}{n}, j=1,2, \ldots, n .\right\},
$$

where $L$ is a positive constant.

We next state the following lemma which will be used in subsequent sections.

Lemma 3 ([10]). Let $x \in P_{T}$. Then $\left|x_{j}^{\sigma}\right|_{0}$ exists and $\left|x_{j}^{\sigma}\right|_{0}=\left|x_{j}\right|_{0}$.

In this paper we assume that $h_{j}$, is continuous, $a_{j} \in \mathcal{R}^{+}$is continuous, $a_{j}(t)>0$ for all $t \in \mathbb{T}$ and

$$
a_{j}(t+T)=a_{j}(t), \quad(i d-r)(t+T)=(i d-r)(t),
$$

where, $i d$ is the identity function on $\mathbb{T}$. We also require that $q_{j}(t, x)$ and $g_{j}(t, x, y)$ are continuous and periodic in $t$ and Lipschitz continuous in $x$ and $y$. That is,

$$
q_{j}(t+T, x)=q_{j}(t, x), g_{j}(t+T, x, y)=g_{j}(t, x, y),
$$

and there are positive constants $E_{1}, E_{2}, E_{3}$ such that

$$
\left|q_{j}(t, x)-q_{j}(t, y)\right| \leq E_{1}|x-y|_{0}, \text { for } x, y \in \mathbb{R},
$$

and

$$
\left|g_{j}(t, x, y)-g_{j}(t, z, w)\right| \leq E_{2}|x-z|_{0}+E_{3}|y-w|_{0}, \text { for } x, y, z, w \in \mathbb{R} .
$$

For our next lemma we consider the neutral dynamic equation

$$
\begin{aligned}
x^{\Delta}(t)= & -a_{j}(t) h_{j}\left(x(\sigma(t))+\left(q_{j}(t, x(t-r(t)))\right)\right)^{\Delta} \\
& +g_{j}(t, x(t), x(t-r(t))), t \in \mathbb{T}, j=1,2, \ldots, n .
\end{aligned}
$$

Lemma 4. Suppose (3.1), (3.2) hold. If $x \in P_{T}$, then $x$ is a solution of equation (3.5) if and only if,

$$
\begin{aligned}
x(t)= & q_{j}(t, x(t-r(t)))+\left(1-e_{\ominus a_{j}}(t, t-T)\right)^{-1} \\
& \times \int_{t-T}^{t}\left[a_{j}(s)\left[x^{\sigma}(s)-h_{j}(x(\sigma(s)))\right]-a_{j}(s) q_{j}^{\sigma}(s, x(s-r(s)))\right. \\
& \left.+g_{j}(s, x(s), x(s-r(s)))\right] e_{\ominus a_{j}}(t, s) \Delta s .
\end{aligned}
$$


Proof. Let $x \in P_{T}$ be a solution of (3.5). First we write (3.5) as

$$
\begin{aligned}
\left\{x(t)-q_{j}(t, x(t-g(t)))\right\}^{\Delta}= & -a_{j}(t)\left\{x^{\sigma}(t)-q_{j}^{\sigma}(t, x(t-r(t)))\right\} \\
& +a_{j}(t)\left[x^{\sigma}(t)-h_{j}(x(\sigma(t)))\right] \\
& -a_{j}(t) q_{j}^{\sigma}(t, x(t-r(t)))+g_{j}(t, x(t), x(t-r(t))) .
\end{aligned}
$$

Multiply both sides by $e_{a_{j}}(t, 0)$ and then integrate from $t-T$ to $t$ to obtain

$$
\begin{aligned}
& \int_{t-T}^{t}\left[e_{a_{j}}(s, 0)\left\{x(s)-q_{j}(s, x(s-r(s)))\right\}\right]^{\Delta} \Delta s \\
& =\int_{t-T}^{t}\left[a_{j}(s)\left[x^{\sigma}(s)-h_{j}(x(\sigma(s)))\right]-a_{j}(s) q_{j}^{\sigma}(s, x(s-r(s)))\right. \\
& \left.\quad+g_{j}(s, x(s), x(s-r(s)))\right] e_{a_{j}}(s, 0) \Delta s .
\end{aligned}
$$

Consequently, we have

$$
\begin{aligned}
& e_{a_{j}}(t, 0)\left(x(t)-q_{j}(t, x(t-r(t)))\right) \\
& \quad-e_{a_{j}}(t-T, 0)\left(x(t-T)-q_{j}(t-T, x(t-T-r(t-T)))\right) \\
& =\int_{t-T}^{t}\left[a_{j}(s)\left[x^{\sigma}(s)-h_{j}(x(\sigma(s)))\right]-a_{j}(s) q_{j}^{\sigma}(s, x(s-r(s)))\right. \\
& \left.\quad+g_{j}(s, x(s), x(s-r(s)))\right] e_{a_{j}}(s, 0) \Delta s .
\end{aligned}
$$

After making use of (3.1), (3.2) and $x \in P_{T}$, we divide both sides of the above equation by $e_{a_{j}}(t, 0)$ to obtain

$$
\begin{aligned}
x(t)= & q_{j}(t, x(t-r(t)))+\left(1-e_{\ominus a_{j}}(t, t-T)\right)^{-1} \\
& \times \int_{t-T}^{t}\left[a_{j}(s)\left[x^{\sigma}(s)-h_{j}(x(\sigma(s)))\right]-a_{j}(s) q_{j}^{\sigma}(s, x(s-r(s)))\right. \\
& \left.+g_{j}(s, x(s), x(s-r(s)))\right] e_{\ominus a_{j}}(t, s) \Delta s .
\end{aligned}
$$

Since each step is reversible, the converse follows. This completes the proof.

Let $\rho(t, t-T)=\operatorname{diag}\left[\rho_{1}, \rho_{2}, \ldots, \rho_{n}\right]$ where $\rho_{j}=\left(1-e_{\ominus a_{j}}(t, t-T)\right)^{-1}$ for $j=$ $1,2, \ldots, n$. Also, we let $\mu(t, s)=\operatorname{diag}\left[e_{\ominus} a_{1}(t, s), \ldots, e_{\ominus a_{n}}(t, s)\right]$.

Define the mapping $F: P_{T} \rightarrow P_{T}$ by

$$
\begin{aligned}
& (F \varphi)(t)=Q(t, \varphi(t-g(t)))+\rho(t, t-T) \int_{t-T}^{t} \mu(t, s) \\
& {\left[A(s)\left[\varphi^{\sigma}(s)-H(\varphi(\sigma(s)))\right]-A(s) Q^{\sigma}(s, \varphi(s-g(s)))+G(s, \varphi(s), \varphi(s-g(s)))\right] \Delta s .}
\end{aligned}
$$


We express equation (3.7) as

$$
(F \varphi)(t)=(B \varphi)(t)+(A \varphi)(t)
$$

where, $A, B$ are given by

$$
(B \varphi)(t)=\rho(t, t-T) \int_{t-T}^{t} \mu(t, s) A(s)\left[\varphi^{\sigma}(s)-H(\varphi(\sigma(s)))\right] \Delta s .
$$

and

$$
\begin{aligned}
& (A \varphi)(t)=Q(t, \varphi(t-g(t))) \\
& +\rho(t, t-T) \int_{t-T}^{t} \mu(t, s)\left[-A(s) Q^{\sigma}(s, \varphi(s-g(s)))+G(s, \varphi(s), \varphi(s-g(s)))\right] \Delta s .
\end{aligned}
$$

In the rest of the section we require the following conditions.

$$
\begin{gathered}
E_{1} \frac{L}{n}+\left|q_{j}(t, 0)\right|_{0} \leq \alpha \frac{L}{n} \\
E_{2} \frac{L}{n}+E_{2} \frac{L}{n}+\left|g_{j}(t, 0,0)\right|_{0} \leq \frac{L}{n} \gamma a_{j}(t),
\end{gathered}
$$

and

$$
J(2 \alpha+\gamma) \leq 1,
$$

where $\alpha, \gamma, L$ and $J$ are constants with $J \geq 3$.

Lemma 5. Suppose (3.1)-(3.4) and (3.10)-(3.12) hold. Then $A: \mathbb{M} \rightarrow \mathbb{M}$, as defined by (3.9), is continuous in the supremum norm and maps $\mathrm{M}$ into a compact subset of $\mathrm{M}$.

Proof. We first show that $A: \mathrm{M} \rightarrow \mathrm{M}$. Evaluate (3.9) at $t+T$.

$$
\begin{aligned}
(A \varphi)(t+T)= & Q(t+T, \varphi(t+T-g(t+T))) \\
& +\rho(t+T, t) \int_{t}^{t+T} \mu(t+T, s)\left[-A(s) Q^{\sigma}(s, \varphi(s-r(s)))\right. \\
& +G(s, \varphi(s), \varphi(s-r(s)))] \Delta s .
\end{aligned}
$$

With $u=s-T$ and using conditions (3.1) - (3.2) we obtain

$$
\begin{aligned}
(A \varphi)(t+T)= & Q(t, \varphi(t-r(t)))+\rho(t+T, t) \\
& \times \int_{t-T}^{t} \mu(t+T, u+T)\left[-A(u+T) Q^{\sigma}(u-T, \varphi(u-T-r(u-T)))\right. \\
& +G(s, \varphi(u-T), \varphi(u-T-r(u-T)))] \Delta u .
\end{aligned}
$$


But we have that $e_{\ominus a_{j}}(t+T, u+T)=e_{\ominus a_{j}}(t, u)$ thus, $\mu(t+T, u+T)=\mu(t, u)$. Moreover, $e_{\ominus a_{j}}(t+T, t)=e_{\ominus a_{j}}(t, t-T)$ and so $\rho(t+T, t)=\rho(t, t-T)$. Thus (3.13) becomes

$$
\begin{aligned}
(A \varphi)(t+T)= & Q(t, \varphi(t-r(t)))+\rho(t, t-T) \\
& \times \int_{t-T}^{t} \mu(t, u)\left[-A(u) Q^{\sigma}(u, \varphi(u-r(u)))\right. \\
& +G(u, \varphi(u), \varphi(u-r(u)))] \Delta u \\
= & (A \varphi)(t) .
\end{aligned}
$$

Note that in view of (3.3) and (3.4) we have that

$$
\begin{aligned}
\left|q_{j}(t, x)\right| & =\left|q_{j}(t, x)-q_{j}(t, 0)+q_{j}(t, 0)\right| \\
& \leq\left|q_{j}(t, x)-q_{j}(t, 0)\right|+\left|q_{j}(t, 0)\right| \\
& \leq E_{1}|x|_{0}+\left|q_{j}(t, 0)\right|_{0} .
\end{aligned}
$$

Similarly,

$$
\begin{aligned}
\left|g_{j}(t, x, y)\right| & =\left|g_{j}(t, x, y)-g_{j}(t, 0,0)+g_{j}(t, 0,0)\right| \\
& \leq\left|g_{j}(t, x, y)-g_{j}(t, 0,0)\right|+\left|g_{j}(t, 0,0)\right| \\
& \leq E_{2}|x|_{0}+E_{3}|y|_{0}+\left|g_{j}(t, 0,0)\right|_{0} .
\end{aligned}
$$

Thus, for any $\varphi \in \mathbb{M}$ we have

$$
\|(A \varphi)\|=\sum_{j=1}^{n} \sup _{t \in[0, T]}\left|\left(A_{j} \varphi\right)(t)\right|
$$

But

$$
\begin{aligned}
& \left|\left(A_{j} \varphi\right)(t)\right|=\mid q_{j}(t, \varphi(t-g(t)))+\left(1-e_{\ominus a_{j}}(t, t-T)\right)^{-1} \\
& \quad \times \int_{t-T}^{t}\left[-a_{j}(s) q_{j}^{\sigma}(s, \varphi(s-r(s)))+g_{j}(s, \varphi(s), \varphi(s-r(s)))\right] e_{\ominus a_{j}}(t, s) \Delta s \mid \\
& \leq\left|q_{j}(t, \varphi(t-r(t)))\right|+\left(1-e_{\ominus a_{j}}(t, t-T)\right)^{-1} \int_{t-T}^{t}\left|-a_{j}(s)\right|\left|q_{j}^{\sigma}(s, \varphi(s-r(s)))\right| \\
& \quad+\left|g_{j}(s, \varphi(s), \varphi(s-r(s)))\right| e_{\ominus a_{j}}(t, s) \Delta s \\
& \leq E_{1} \frac{L}{n}+\left|q_{j}(t, 0)\right|_{0}+\left(1-e_{\ominus a_{j}}(t, t-T)\right)^{-1} \\
& \quad \times \int_{t-T}^{t}\left[a_{j}(s)\left(E_{1} \frac{L}{n}+\left|q_{j}(s, 0)\right|_{0}\right)+\left(E_{2}+E_{3}\right) \frac{L}{n}+\left|g_{j}(s, 0,0)\right|_{0}\right] e_{\ominus a_{j}}(t, s) \Delta s \\
& \leq \alpha \frac{L}{n}+\left(1-e_{\ominus a}(t, t-T)\right)^{-1}
\end{aligned}
$$




$$
\begin{aligned}
& \times \int_{t-T}^{t}\left[\alpha \frac{L}{n}+\gamma \frac{L}{n}\right] a(s) e_{\ominus a}(t, s) \Delta s \\
\leq & (2 \alpha+\gamma) \frac{L}{n} \leq \frac{L}{n J} .
\end{aligned}
$$

Thus,

$$
\|(A \varphi)\| \leq \sum_{j=1}^{n} \frac{L}{n J} \leq \frac{L}{J}<L,
$$

showing that $A$ maps $\mathbb{M}$ into itself. To see that $A$ is continuous, let $\varphi, \psi \in \mathbb{M}$ and define

$$
\begin{aligned}
\eta & :=\sup _{t \in[0, T]}\left|\left(1-e_{\ominus a_{j}}(t, t-T)\right)^{-1}\right|, \sigma:=\sup _{t \in[0, T]}\left|a_{j}(t)\right|, \\
\gamma & \left.:=\sup _{u \in[t-T, t]} e_{\ominus a_{j}}(t, u), \lambda:=\sup _{t \in[0, T]} \mid\left(q_{j}(t, x(t), x(t-r(t)))\right)\right)^{\Delta} \mid, \\
\alpha & :=\sup _{t \in[0, T]}\left|q_{j}(t, 0)\right|, \quad \beta:=\sup _{t \in[0, T]}\left|g_{j}(t, 0,0)\right| .
\end{aligned}
$$

Given $\varepsilon>0$, take $\delta=\varepsilon / n M$ with $M=E_{1}+\eta \gamma T\left(\sigma E_{1}+E_{2}+E_{3}\right)$ where, $E_{1}$, $E_{2}$ and $E_{3}$ are given in (3.3) and (3.4) such that $\|\varphi-\psi\|<\delta$. Using (3.9) we get

$$
\|A \varphi-A \psi\|=\sum_{j=1}^{n} \sup _{t \in[0, T]}\left|\left(A_{j} \varphi\right)(t)-\left(A_{j} \psi\right)(t)\right| .
$$

But,

$$
\begin{aligned}
\left|A_{j} \varphi-A_{j} \psi\right|_{0} & \leq E_{1}|\varphi-\psi|_{0}+\eta \gamma \int_{0}^{T}\left[\sigma E_{1}|\varphi-\psi|_{0}+\left(E_{2}+E_{3}\right)|\varphi-\psi|_{0}\right] \Delta u \\
& \leq M|\varphi-\psi|_{0} .
\end{aligned}
$$

Thus,

$$
\|A \varphi-A \psi\| \leq n M\|\varphi-\psi\|<\varepsilon .
$$

This proves that $A$ is continuous.

We next show that $A$ is compact. Consider the sequence of periodic functions $\left\{\varphi_{n}\right\} \subset \mathbb{M}$. Thus as before we have that

$$
\left\|A\left(\varphi_{n}\right)\right\| \leq L,
$$

showing that the sequence $\left\{A \varphi_{n}\right\}$ is uniformly bounded. Now, it can be easily checked that

$$
\begin{gathered}
\left.\left(A_{j} \varphi_{n}\right)^{\Delta}(t)=\left(q_{j}\left(t, \varphi_{n}(t), \varphi_{n}(t-r(t))\right)\right)\right)^{\Delta}-a_{j}(t) q_{j}^{\sigma}\left(t, \varphi_{n}(t-r(t))\right) \\
\quad+g_{j}\left(t, \varphi_{n}(t), \varphi_{n}(t-r(t))\right)-a_{j}(t)\left\{\left(1-e_{\ominus a}(t, t-T)\right)^{-1}\right.
\end{gathered}
$$




$$
\begin{gathered}
\left.\quad \times \int_{t-T}^{t}\left[-a_{j}(s) q_{j}^{\sigma}\left(s, \varphi_{n}(s-r(s))\right)+g_{j}\left(s, \varphi_{n}(s), \varphi_{n}(s-r(s))\right)\right] e_{\ominus a}(t, s) \Delta s\right\} \\
\left.=\left(q_{j}\left(t, \varphi_{n}(t), \varphi_{n}(t-r(t))\right)\right)\right)^{\Delta}-a_{j}(t) q_{j}^{\sigma}\left(t, \varphi_{n}(t-r(t))\right) \\
+g_{j}\left(t, \varphi_{n}(t), \varphi_{n}(t-r(t))\right)-a_{j}(t)\left\{\left(1-e_{\ominus a}(t, t-T)\right)^{-1}\right. \\
\times \int_{t-T}^{t}\left[-a_{j}(s) q_{j}^{\sigma}\left(s, \varphi_{n}(s-r(s))\right)+g_{j}\left(s, \varphi_{n}(s), \varphi_{n}(s-r(s))\right)\right] e_{\ominus a}(t, s) \Delta s \\
\left.+q_{j}\left(t, \varphi_{n}(t-r(t))\right)\right\}+a_{j}(t) q_{j}(t, \varphi(t-r(t))) . \\
\left.\quad\left(A_{j} \varphi_{n}\right)^{\Delta}(t)=\left(q_{j}\left(t, \varphi_{n}(t), \varphi_{n}(t-r(t))\right)\right)\right)^{\Delta} \\
\quad-a_{j}(t)\left(A_{j} \varphi_{n}\right)^{\sigma}(t)-a_{j}(t) q_{j}^{\sigma}\left(t, \varphi_{n}(t-r(t))\right) \\
+g_{j}\left(t, \varphi_{n}(t), \varphi_{n}(t-r(t))\right)+a_{j}(t) q_{j}\left(t, \varphi_{n}(t-r(t))\right) .
\end{gathered}
$$

Consequently,

$$
\left|\left(A_{j} \varphi_{n}\right)^{\Delta}(t)\right| \leq \lambda+\sigma L+2 \sigma\left(E_{1} \frac{L}{n}+\alpha\right)+E_{2} \frac{L}{n}+E_{3} \frac{L}{n}+\beta
$$

for all $n$.

Thus,

$$
\left\|\left(A \varphi_{n}\right)^{\Delta}\right\| \leq \sum_{j=1}^{n}\left(\lambda+\sigma L+2 \sigma\left(E_{1} \frac{L}{n}+\alpha\right)+E_{2} \frac{L}{n}+E_{3} \frac{L}{n}+\beta\right)=F .
$$

That is $\left\|\left(A \varphi_{n}\right)^{\Delta}\right\| \leq F$, for some positive constant $F$. Thus the sequence $\left\{A \varphi_{n}\right\}$ is uniformly bounded and equi-continuous. The Arzela-Ascoli theorem implies that there is a subsequence $\left\{A \varphi_{n_{k}}\right\}$ which converges uniformly to a continuous $T$-periodic function $\varphi^{*}$. Thus A is compact.

We next state the following proposition (see [1]), in which the following assumptions are made on the function $h: \mathbb{R} \rightarrow \mathbb{R}$.

(H1) $h$ is continuous on $U_{l}=[-l, l]$ and differentiable on $U_{l}$.

(H2) $h$ is strictly increasing on $U_{l}$.

(H3) $\sup _{s \in U_{l}} h^{\Delta}(s) \leq 1$.

Proposition 1 ([1]). Let h be a function satisfying $(H 1)-(H 3)$. Then the mapping $\mathfrak{h}(\varphi)(t)=\varphi(t)-h(\varphi(t))$ is a large contraction on the set $\mathbf{M}_{l}$.

The next result gives a relationship between the mappings $\mathfrak{h}_{j}$ and $B$ in the sense of large contraction.

Lemma 6. If $\mathfrak{h}_{\mathrm{j}}$ is a large contraction on $\mathrm{M}$, then so is the mapping $B$. 
Proof. If $\mathfrak{h}_{\mathrm{j}}$ is a large contraction on $\mathbb{M}$, then for $x, y \in \mathbb{M}$, with $x \neq y$, we have $\left\|\mathfrak{h}_{\mathfrak{j}} x-\mathfrak{h}_{\mathfrak{j}} y\right\| \leq|x-y|_{0}$. Then it follows from the equality

$$
a_{j}(u) e_{\ominus a_{j}}(t+T, \sigma(u))=\left[e_{\ominus a_{j}}(t+T, u)\right]^{\Delta_{s}},
$$

where $\Delta_{s}$ indicates the delta derivative with respect to $s$ that

$$
\begin{aligned}
\left|B_{j} x(t)-B_{j} y(t)\right| & \leq \int_{t}^{t+T} \frac{e_{\ominus a_{j}}(t+T, \sigma(u))}{1-e_{\ominus a_{j}}(t, t+T)} a_{j}(u)\left|\mathfrak{h}_{\mathbf{j}}(x(u))-\mathfrak{h}_{j}(y(u))\right| \Delta u \\
& \leq \frac{|x-y|_{0}}{1-e_{\ominus a_{j}}(t, t+T)} \int_{t}^{t+T} a_{j}(u) e_{\ominus a_{j}}(t+T, \sigma(u)) \Delta u \\
& =|x-y|_{0} .
\end{aligned}
$$

Thus,

$$
\begin{aligned}
\|B x-B y\| & =\sum_{j=1}^{n} \sup _{t \in[0, T]}\left|B_{j} x(t)-B_{j} y(t)\right| \\
& \leq \sum_{j=1}^{n}|x-y|_{0}=\|x-y\|
\end{aligned}
$$

One may also show in a similar way that

$$
\|B x-B y\| \leq \delta\|x-y\|
$$

holds if we know the existence of a $0<\delta<1$, such that for all $\epsilon>0$

$$
[x, y \in \mathbb{M},\|x-y\| \geq \epsilon] \Rightarrow\|B x-B y\| \leq \delta\|x-y\| .
$$

The proof is complete.

Lemma 7. Suppose (3.1)-(3.4), and (3.10)-(3.12) hold. Suppose also that

$$
\max \left(\left|\mathfrak{h}_{\mathbf{j}}(-L)\right|,\left|\mathfrak{h}_{\mathbf{j}}(L)\right|\right) \leq \frac{(J-1) L}{J n} .
$$

For $B, A$ defined by (3.8) and (3.9), if $\varphi, \psi \in \mathbb{M}$ are arbitrary, then

$$
A \varphi+B \psi: \mathbb{M} \rightarrow \mathbb{M} .
$$

Proof. Let $\varphi, \psi \in \mathbb{M}$ be arbitrary. Using the definition of $B$ and the result of Lemma 5 we obtain

$$
\begin{aligned}
\left\|A_{j}(\varphi)+B_{j}(\psi)\right\| \leq & \left|q_{j}(t, \varphi(t-r(t)))\right| \\
& +\left(1-e_{\ominus a_{j}}(t, t-T)\right)^{-1} \int_{t-T}^{t}\left|-a_{j}(s)\right|\left|q_{j}^{\sigma}(s, \varphi(s-r(s)))\right| \\
& +\left|g_{j}(s, \varphi(s), \varphi(s-r(s)))\right| e_{\ominus a_{j}}(t, s) \Delta s \\
& +\max \left(\left|\mathfrak{h}_{j}(-L)\right|,\left|\mathfrak{h}_{j}(L)\right|\right) \int_{t}^{t+T} \frac{e_{\ominus a_{j}}(t+T, \sigma(u))}{1-e_{\ominus a_{j}}(t, t+T)} a_{j}(u) \Delta u
\end{aligned}
$$




$$
\leq \frac{L}{J n}+\frac{(J-1) L}{J n}=\frac{L}{n}
$$

Thus,

$$
\|A(\varphi)+B(\psi)\| \leq \sum_{j=1}^{n} \frac{L}{n}=L .
$$

This completes the proof.

Theorem 4. Suppose (3.1)-(3.4) and (3.10)-(3.12) hold. Suppose further that the hypotheses of Lemma 5, Lemma 6 and Lemma 7 hold. Then equation (1.1) has a periodic solution in the subset $\mathrm{M}$.

Proof. By Lemma 5, $A: \mathbb{M} \rightarrow \mathbb{M}$ is completely continuous. By Lemma 7, $A \varphi+$ $B \psi \in \mathbb{M}$ whenever $\varphi, \psi \in \mathbb{M}$. Moreover, $B: \mathbb{M} \rightarrow \mathbb{M}$ is a large contraction by Lemma 6 . Thus all the hypotheses of Theorem 3 are satisfied. Thus, there exists a fixed point $\varphi \in \mathbb{M}$ such that $\varphi=A \varphi+B \varphi$. Hence (1.1) has a $T$ - periodic solution.

\section{REFERENCES}

[1] M. Adivar and Y. Raffoul, "Existence of periodic solutions in totally nonlinear delay dynamic equations," Elect. J. Qual. Theo. Diff. Equ., vol. 2009, no. 1, pp. 1-20, 2009, doi: 10.14232/ejqtde.2009.4.1.

[2] A. Ardjouni and A. Djoudi, "Periodic solutions in totally nonlinear dynamic equations with functional delay on a time scale," Rend. Sem. Mat. Univ. Politec. Torino, vol. 68, no. 4, pp. 349-359, 2010.

[3] A. Ardjouni and A. Djoudi, "Existence of periodic solutions for nonlinear neutral dynamic equations with variable delay on a time scale," Commun. Nonlinear Sci. Numer. Simulat., vol. 17, pp. 3061-3069, 2012, doi: 10.1016/j.cnsns.2011.11.026.

[4] A. Ardjouni and A. Djoudi, "Existence of periodic solutions for nonlinear neutral dynamic equations with functional delay on a time scale," Acta Univ. Palacki. Olomuc. Fac. rer. nat. Mathematica, vol. 52, no. 2, pp. 5-19, 2013.

[5] A. Ardjouni and A. Djoudi, "Existence of positive periodic solutions for nonlinear neutral dynamic equations with variable delay on a time scale," Malaya J. Matematik, vol. 2, no. 1, pp. 60-67, 2013.

[6] F. Atici, G. Guseinov, and B. Kaymakcalan, "Stability criteria for dynamic equations on time scales with periodic coefficients," in Proceedings of the International Conference on Dynamic Systems and Applications, ser. III. International Conference, 1999, pp. 43-48.

[7] M. Bohner and A. Peterson, Dynamic Equations on Time Scales, An introduction with Applications. Boston: Birkhäuser, 2001. doi: 10.1007/978-1-4612-0201-1.

[8] M. Bohner and A. Peterson, Advances in Dynamic Equations on Time Scales. Boston: Birkhäuser, 2003. doi: 10.1007/978-0-8176-8230-9.

[9] T. Burton, Stability by fixed point theory for functional differential equations, 2nd ed., ser. Series is books. New York: Dover, 2006, vol. III.

[10] E. Kaufmann and Y. Raffoul, "Periodic solutions for a neutral nonlinear dynamical equation on a time scale," J. Math. Anal. Appl., vol. 319, no. 1, pp. 315-325, 2006.

[11] E. Kaufmann and Y. Raffoul, "Periodicity and stability in neutral nonlinear dynamic equations with functional delay on a time scale," Electron. J. Differential Equations, vol. 2007, no. 27, pp. 1-12, 2007. 
[12] E. Kaufmann and Y. Raffoul, "Stability in neutral nonlinear dynamic equations on a time scale with functional delay," Electronic Journal of Qualitative Theory of Differential Equations, vol. 2009, no. 1, pp. 1-20, 2009, doi: 10.1016/j.jmaa.2006.01.063.

\section{Author's address}

\section{Ernest Yankson}

University of Cape Coast, Mathematics and Statistics Department, Cape Coast, Ghana

E-mail address: ernestoyankegmail.com 